\title{
Variation of $\sum \mathfrak{3}$ and Coherent $\sum 3$ Boundary Fraction with Thickness in Nanometric Cu Films
}

\author{
X. Liu ${ }^{1}$, A. P. Warren ${ }^{2}$, T. N. Nuhfer ${ }^{1}$, G.S. Rohrer ${ }^{1}$, K. R. Coffey ${ }^{2}$, K. Barmak ${ }^{1,3}$ \\ ${ }^{1}$ Materials Research Science and Engineering Center, and the Department of Materials Science and \\ Engineering, Carnegie Mellon University, Pittsburgh, USA \\ ${ }^{2}$ Department of Materials Science and Engineering, , University of Central Florida, 4000 Central \\ Florida Boulevard, Orlando, USA \\ ${ }^{3}$ Department of Applied Physics and Applied Mathematics, Columbia University, 500 W. 120 Street, \\ New York, USA
}

Twin boundaries with a $60^{\circ}$ misorientation about $<111>$ play a significant role in the properties of nanocrystalline $\mathrm{Cu}$ [1]. This misorientation is designated as $\sum 3$ in the coincident site lattice (CSL) notation. A $\sum 3$ boundary is classified as coherent when the boundary plane is $\{111\}$. In this study, $\sum 3$ as well as coherent $\sum 3$ boundary length fractions in a set of six heavily-twinned $\mathrm{Cu}$ films were quantified using a transmission electron microscope (TEM) based orientation mapping tool. The films had thicknesses in the range of 25.6-111.1 nm. The films were sputter deposited as a stack of $\mathrm{SiO}_{2}(2 \mathrm{~nm}) / \mathrm{Ta}_{38} \mathrm{Si}_{14} \mathrm{~N}_{48}(2 \mathrm{~nm}) / \mathrm{Cu} / \mathrm{Ta}_{38} \mathrm{Si}_{14} \mathrm{~N}_{48}(2 \mathrm{~nm}) / \mathrm{SiO}_{2}(2 \mathrm{~nm})$ onto oxidized Si substrates at $40^{\circ} \mathrm{C}$ and were annealed at $600^{\circ} \mathrm{C}$ for 30 minutes in an $\mathrm{Ar}+3 \% \mathrm{H}_{2}$ atmosphere following deposition. The TEM orientation maps were obtained using the ASTAR system developed by NanoMEGAS [2]. The orientation of each point in the field of view was determined by cross-correlation of precalculated diffraction patterns (templates) with observed spot diffraction patterns. Electron microscopy was performed on a $200 \mathrm{kV}$ Tecnai F20 field emission TEM. Precession angles ranging between $0.3^{\circ}$ and $0.5^{\circ}$ were used to reduce the strong dynamical effects and to average out the small local orientation changes in a given region [3]. The spot size for the scans was approximately $1 \mathrm{~nm}$. The step size for the scans was increased from $2.5 \mathrm{~nm}$ for the sample with the smallest layer thickness $(25.6 \mathrm{~nm})$ to $12.5 \mathrm{~nm}$ for the sample with the largest thickness $(111.1 \mathrm{~nm})$. An example crystal orientation map in the sample normal direction is shown in Fig. 1(a). Figure 1(b) gives the virtual bright field image of the same field of view as in Fig. 1(a). The virtual bright field image is obtained by placing a virtual aperture around the transmitted beam and recording the variation in the intensity of this beam. The index map in Fig. 1(c) gives a grayscale representation of the correlation index between the diffraction pattern and the best matching template.

The grain sizes of the samples were determined for statistically significant populations $(>1000$ grains/per sample)[4] using both the conical dark field imaging in the Automated Crystallography in the TEM (ACT) system and the ASTAR orientation mapping system. Grain sizes, as equivalent circle diameter of mean area, were found to be 51 to $315 \mathrm{~nm}$. The grain size values for the two techniques were found to agree well (Table 1) for a suitable choice of cleanup parameters for the orientation maps. The $\sum 3$ boundary fraction, as multiples of random distribution (MRD), was obtained via stereological analysis of more than 50,000 boundary line segments per sample [5]. $\sum 3$ and coherent $\sum 3$ boundaries can also be reconstructed using the TSL ${ }^{\mathrm{TM}}$ software, examples of which are shown in Figure 2. The length fractions of $\sum 3$ and coherent $\sum 3$ boundaries were found to increase monotonically with grain size (Figure 3 ). The grain boundary character distribution (GBCD) gives the relative area in 3D and relative length in 2D of the boundaries with a given misorientation and boundary normal, with values greater than one MRD indicating that the grain boundary type occurs more frequently than would be expected in a random distribution. The GBCD values for the $\sum 3$ boundaries given in the table are seen to be $>>1$ and to increase with film thickness. 


\section{References:}

[1] K. Lu, L. Lu and S. Suresh, Science, 324 (1998) 555.

[2] E. Rauch and L. Dupuy, Arch Metall Mater 50 (2005) 87.

[3] E. Rauch et al., Micros. Anal 93 (2008) 5.

[4] D. Carpenter, J. Rickman and K. Barmak, J. Appl. Phys, 84 (1998) 5843.

[5] D. M. Saylor et al., Metall. Mater. Trans. A, 35 (2004) 1981.

[6] Financial support of the Semiconductor Research Corporation, Task 2121.001 is gratefully acknowledged.

Table of film thickness, $\sum 3$ twin boundary analysis and grain size of $\mathrm{Cu}$ films. The grain boundary character distribution (GBCD) is given in multiples of random distribution (MRD).

\begin{tabular}{|c|c|c|c|c|c|}
\hline $\begin{array}{c}\text { Film Thickness } \\
(\mathrm{nm})\end{array}$ & $\begin{array}{c}\text { Twin Fraction } \\
(\%)\end{array}$ & $\begin{array}{c}\text { Coherent Fraction } \\
(\%)\end{array}$ & $\begin{array}{c}\text { GBCD } \\
(\mathrm{MRD})\end{array}$ & $\begin{array}{c}\text { Grain size } \\
(\mathrm{nm})\end{array}$ & $\begin{array}{c}\text { Grain size }^{2} \\
(\mathrm{~nm})\end{array}$ \\
\hline 25.6 & $41 \pm 5$ & $43 \pm 5$ & 970 & 52 & 51 \\
\hline 38.2 & $43 \pm 3$ & $47 \pm 5$ & 1160 & 75 & 69 \\
\hline 39.3 & $42 \pm 3$ & $50 \pm 5$ & 1110 & 90 & 87 \\
\hline 56.8 & $44 \pm 3$ & $51 \pm 5$ & 1180 & 112 & 106 \\
\hline 72.4 & $48 \pm 4$ & $51 \pm 5$ & 1440 & 156 & 157 \\
\hline 110 & $53 \pm 4$ & $56 \pm 11$ & 1570 & 315 & 315 \\
\hline \multicolumn{2}{|r|}{ Grain size } & is the ACT grain size while grain size $^{2}$ is the ASTAR orientation mapping grain size \\
\hline
\end{tabular}
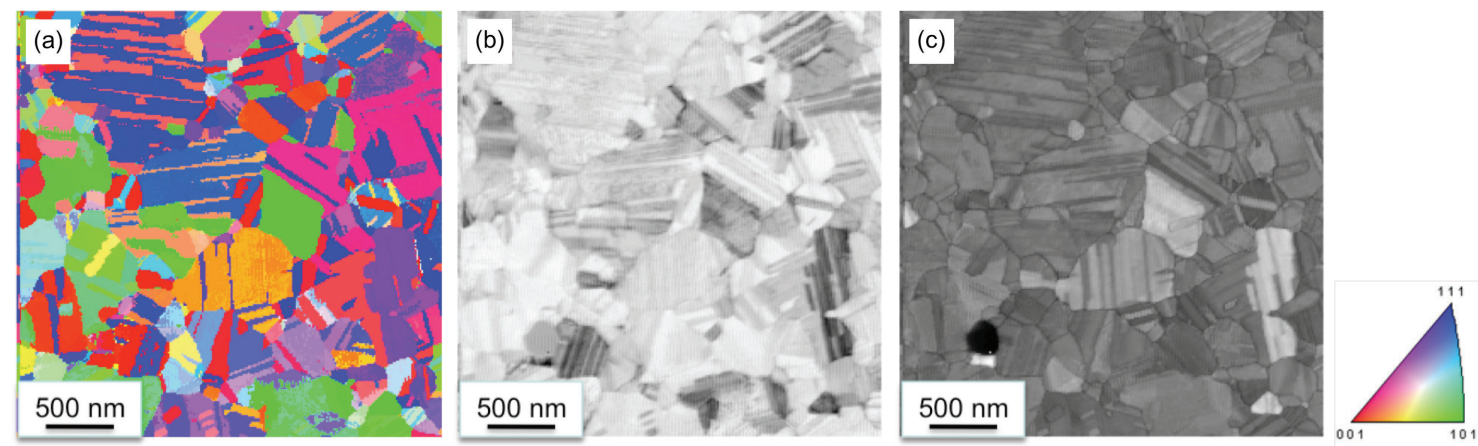

Figure 1. (a) The crystal orientation map given as the inverse pole figure map along the sample normal (Z) direction, (b) the virtual bright field image, and (c) the correlation index map for the $111.1 \mathrm{~nm}$-thick $\mathrm{Cu}$ film. The key for the orientation map is given on the right.
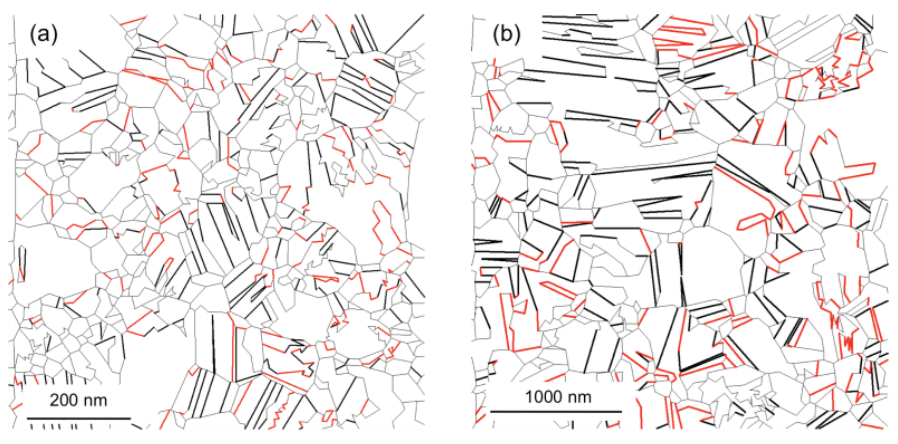

Figure 2. Trace analysis of Cu films (a) $35 \mathrm{~nm}$, and (b) $110 \mathrm{~nm}$ thick. Thick Thick black lines represent coherent $\sum 3$; thick red lines represent incoherent $\sum 3$; thin black lines represent other boundary types.

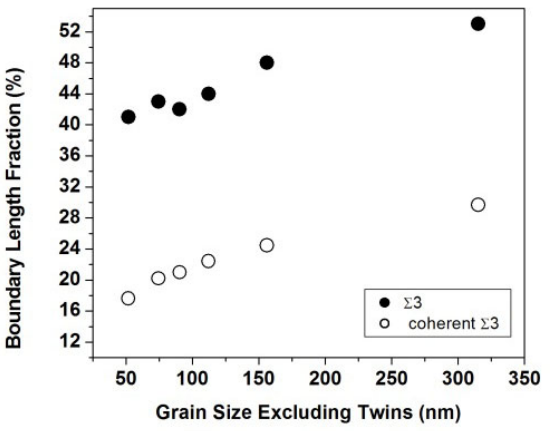

Figure 3. $\sum 3$ and coherent $\sum 3$ fraction versus grain size. 